\title{
MOUVEMENT ET CRISTALLISATIONS : L'ACTUEL ENTRE MYTHOLOGIE, CINÉMA ET THÉORIE DU WEB
}

Peppe Cavallari et Marcello Vitali-Rosati

\author{
Association Sens-Public | « Cahiers Sens public »
}

2018/1 n²1-22 | pages 33 à 54

ISSN $1767-9397$

ISBN 9782956602002

Article disponible en ligne à l'adresse :

https://www.cairn.info/revue-cahiers-sens-public-2018-1-page-33.htm

Distribution électronique Cairn.info pour Association Sens-Public.

(C) Association Sens-Public. Tous droits réservés pour tous pays.

La reproduction ou représentation de cet article, notamment par photocopie, n'est autorisée que dans les limites des conditions générales d'utilisation du site ou, le cas échéant, des conditions générales de la licence souscrite par votre établissement. Toute autre reproduction ou représentation, en tout ou partie, sous quelque forme et de quelque manière que ce soit, est interdite sauf accord préalable et écrit de l'éditeur, en dehors des cas prévus par la législation en vigueur en France. Il est précisé que son stockage dans une base de données est également interdit. 


\section{Mouvement et cristallisations : l'actuel entre mythologie, \\ cinéma et théorie du web}

PEPPE CAVALLARI \& MARCELLO VITALI-ROSATI 
Bio Marcello Vitali-Rosati, professeur au département des littératures de langue française de I'Université de Montréal, est titulaire d'une Chaire de recherche du Canada sur les écritures numériques. II mène une réflexion philosophique sur les enjeux des technologies numériques : le concept de virtuel, l'identité numérique, les notions d'auteur et d'autorité, les formes de production, légitimation et circulation du savoir à l'époque du web, et la théorie de l'éditorialisation. Auteur de nombreux articles et monographies, il dirige la revue Sens public et co-dirige la collection «Parcours Numériques» (Presses de l'Université de Montréal). Responsable de plusieurs projets en humanités numériques, particulièrement dans le domaine de l'édition savante, il développe des plateformes d'édition de revues et de monographies enrichies, un logiciel d'édition d'articles scientifiques ainsi qu'une plateforme d'édition collaborative du Codex Palatinus 23.

Bio Peppe Cavallari, doctorant en philosophie (UTC-UDEM) prépare une thèse sur la performativité et les effets de la présence numérique, dispense des cours en Culture numérique à Hétic (Hautes études en Technologie de l'information e de la communication-Montreuil) où il est responsable de la recherche en humanisme numérique et, à l'université Paris-Nanterre. II coordonne un séminaire de culture numérique - \#UVDE - et participe aux travaux du département Humanisme numérique (Collège des Bernardins, Paris) et de la Chaire de Recherche du Canada sur les écritures numériques (UdeM, Montréal). 
Résumé : Le rapport entre actuel et virtuel a été très problématisé dans l'ensemble de l'histoire de la pensée occidentale. À partir de la critique nietzschéenne de l'essentialisme, l'idée aristotélicienne de la primauté ontologique de l'acte (entelecheia) sur la puissance (dunamis) a été profondément mise en question : il n'y aurait pas d'essence, il n'y aurait que des actions. Ainsi, le mouvement, les dynamiques, le processus, auraient une priorité sur leur actualisation en essences qui ne serait qu'un contrecoup de la puissance.

Cette structure de priorité de la dynamique sur l'actualité semble s'adapter particulièrement aux environnements numériques, toujours en mouvement. Pourtant, l'économie des notifications toujours plus nombreuses, ainsi que la permanence et l'enregistrement par défaut de tous nos actes, se révèle indispensable aux fins de la production du mouvement - qui serait en réalité fondé sur l'immobilité. Dans cet article, nous allons revenir sur le rapport entre actuel et virtuel pour souligner l'importance du concept d'actuel.

Mots clés : Actuel - Virtuel - Mouvement - Notifications Enregistrement - Cinéma - Facebook - Web

Abstract: The connection between actual and virtual has been very much problematized in the history of Western thought. Based on Nietzsche criticism of essentialism, the Aristotelian idea of ontological primacy of acting over power was deeply questionned: in fact, the essence would not exist, only actions would. Therefore, the movement, the dynamics, the process would have a priority over their actualisation as essence, and that would be the result of power.

This structure prioritizing dynamic over actuality seems to particularly adapt to digital environnements which are always evolving. However, the growing number of notifications and the constant recording of our actions turns out to be essential to the goals of producing the movement - the latter being actually based on immobility. In this article, we will analyze the connections between actual and virtual to highlight how important the concept of actual is.

Keywords: Current - Virtual - Motion - Notification Recording - Cinema - Facebook - Web 


\section{Le flux et le photogramme}

Cyparissos est un jeune homme de l'île de Cos, amoureux d'un magnifique cerf. Par erreur, un jour, le javelot de Cyparissos tue le cerf. La douleur du jeune homme est infinie: il demande aux dieux de ne pas y survivre. Son sang se tarit, son teint verdit, ses cheveux se hérissent : il se transforme en cyprès.

Pygmalion déteste les femmes et ne veut tomber amoureux d'aucune. Mais il sculpte une statue d'ivoire qui représente une femme. La statue est si belle qu'il en tombe amoureux

36 et la traite comme si elle était une femme vivante. Les dieux exaucent ses prières : un souffle semble commencer à sortir de la bouche de la statue, l'ivoire de ses seins fléchit sous la main, ses veines s'enflent. La statue est devenue une femme.

Les Métamorphoses d'Ovide ${ }^{\top}$ - dont ces deux histoires sont tirées - racontent principalement le passage du mouvement à l'immobilité et de l'immobilité au mouvement. C'est la transformation par excellence, simple et pourtant merveilleuse: une chose qui ne bouge pas se met à bouger, ou une chose qui semble nous échapper par son mouvement s'arrête et tombe dans nos mains.

C'est à la lumière de ce passage que nous allons proposer une interprétation d'un autre couple de concepts dont la relation complexe a traversé I'histoire de la pensée : celui de virtuel et d'actuel. On peut constater que l'idée aristotélicienne d'une primauté de l'acte sur la puissance a été le paradigme de pensée dominant pendant plusieurs siècles.

1. Cf. Ovide. Métamorphoses. Hugo MAGNUS, Gotha (Allemagne): Friedr. Andr. Perthes. 1892. En ligne : http://www.perseus.tufts.edu/hopper/ text?doc=urn:cts:IatinLit:phi0959. phi006. 
L'acte (entelecheia) est supérieur à la puissance (dunamis) parce qu'il est ce vers quoi la puissance tend : il est la cristallisation immobile du mouvement. Sur cette primauté sont basées toutes les théories essentialistes : la primauté de l'acte permet de saisir le monde, de le définir, de le comprendre. On ne peut saisir que ce qui ne change plus ; le mouvement de la puissance nous met devant des choses éphémères, qui glissent entre les mains de celui qui essaie de les comprendre. Or, la critique nietzschéenne de l'essentialisme a profondément mis cette idée en question : il n'y aurait pas d'essence, il n'y aurait que des actions ${ }^{2}$; le mouvement, les dynamiques, le processus, auraient une priorité sur leur actualisation en essences. Au $x x^{e}$ siècle, les philosophies de Bergson ${ }^{3}$ et de Deleuze ${ }^{4}$ montrèrent ainsi l'essence actuelle comme un contrecoup de la puissance.

On pourrait dire que l'actuel, depuis au moins un siècle, n'est pas à la mode. Or, la structure de priorité de la dynamique sur l'actualité semblerait s'adapter particulièrement aux environnements numériques, toujours en mouvement. Pourtant, l'économie des notifications toujours plus nombreuses, ainsi que la permanence et l'enregistrement par défaut de tous nos actes se révèlent indispensables aux fins de la production du mouvement. Le mouvement est en réalité fondé sur l'immobilité. Sans la dernière trace figée et actualisée, on ne pourrait pas relancer de dynamique. Nous voudrions ici revenir sur le rapport entre actuel et virtuel pour souligner l'importance du concept d'actuel : si l'actuel est un contrecoup, il reste un contrecoup nécessaire sans lequel rien de virtuel ne pourrait être conçu.

2. Cf. notamment la Généalogie de la morale : « Mais un tel substrat n'existe pas ; II n'y a pas d'"être" derrière l'agir, la production d'effets, le devenir ; "l'agent" est purement et simplement ajouté de manière imaginative à l'agir - l'agir est tout. », p. 97-98.

3. En particulier Henri BERGSON, L'évolution créatrice. Paris: Presses universitaires de France, 1966.

4. Cf. en particulier Gilles DELEUZE, Différence et répétition. Paris : Presses universitaires de France, 1972. 


\section{La construction du concept}

La possibilité du non-changement est à la base de la structure de notre pensée et de notre langage. Quand on dit le mot « souffrance», on reproduit la métamorphose de Cyparissos. Le flux changeant de la souffrance que vit Cyparissos ne peut pas être dit, car il est en mouvement, il change. Pour dire cette souffrance, il faut imaginer qu'elle reste ce qu'elle est, au moins pendant qu'on la dit. Cette fixation correspond à ce que le jeune homme demande pour pouvoir penser sa douleur: il faut qu'elle reste ce qu'elle est pour qu'on puisse la dire et la comprendre. Le mouvement de la souffrance se cristallise donc : il devient cyprès. C'est là une forme d'actualisation: le flux virtuel, mouvant et insaisissable, doit s'arrêter dans quelque chose de figé pour qu'on puisse le saisir.

La cristallisation désigne justement le processus par lequel le mouvement se transforme en immobilité. La formation des cristaux est une métaphore assez claire de ce processus : les cristaux, résultat immobile d'un mouvement, sont le signe du passage du mouvement. En regardant les cristaux immobiles, on peut deviner le mouvement qui a produit cette immobilité.

La métamorphose de Cyparissos consiste donc en une cristallisation : le mouvement de sa souffrance se fige dans les traces qu'il laisse, comme les larmes qui se muent en résine; le mouvement se transforme en traces, et ces traces restent immobiles. C'est le processus avec lequel nous construisons nos mots et nos concepts, des plus simples aux plus compliqués. Car le même processus de cristallisation et d'actualisation vaut aussi pour un mot - et un concept - comme «chaise».

Le mot «chaise » présuppose que ce qu'est une chaise dans la réalité reste égal à lui-même, au moins pendant qu'on en parle. Nous savons très bien que ce n'est pas ainsi : même la chaise est dans un flux, dans un mouvement. Le bois se modifie, vieillit, sa couleur change, sa forme aussi. Et un autre problème s'ajoute : le mot «chaise » renvoie à toutes les chaises, avec toutes leurs différences. Dans l'unité du mot « chaise », on cristallise cette multiplicité dans le temps et l'espace. C'est comme si l'on prenait un appareil photo avec un 
très long temps de pause, qu'on faisait passer devant l'objectif toutes les chaises possibles et qu'on utilisait la photographie pour signifier le processus entier.

L'anthropologie contemporaine fait du mouvement le facteur à la base de la production de l'espace social : s'inspirant de la leçon d'Henri Lefebvre, Christoph Wulf affirme que

« [l]e mouvement crée le monde dans un échange réciproque continuel; il utilise pour cela la plasticité du corps et la performabilité du monde. Le mouvement est un médium entre le corps et le monde, et c'est par lui qu'ils sont étroitement reliés I'un à l'autre. Par le mouvement, les hommes prennent part aux mondes des autres et chacun d'eux devient ainsi une partie de la communauté humaine ».

Et encore, plus loin dans son essai, il cite Arnold Gehlen :

"L'espace de mouvement contient bien davantage que la seule situation qu'il donne à voir. À celle-ci se superposent tous les mouvements anticipés, non encore réalisés dans l'instant présent, mais dont la possibilité est déjà contenue dans ceux qui sont perçus. Cette anticipation n'est pas d'ordre cognitif, elle n'est pas le fait d'un acte de connaissance, mais elle surgit directement dans la situation: "on voit d'après les mouvements" ce qui va se passer $»^{5}$.

Le mouvement dont il est question ici correspond parfaitement à la notion de performance telle qu'elle a été théorisée par Richard Schechner qui, tout comme Wulf et Lefebvre, par l'adoption d'une acception assez large d'une notion concernant aussi bien les rituels que les sports, en fait la base

5. Christoph WULF, Une anthropologie historique et culturelle. Rituels, mimésis sociale et performativité, trad. fr., Paris : Téraèdre, 2007, p. 85 ; 107. 
fondatrice de notre existence sociale. La fonction de la performance face à la communauté et au réel est double :

\begin{abstract}
«Elle est à la fois régulatrice et perturbatrice. Elle est transmission culturelle - cérémonies, mariages, etc. - et perpétue ou entérine les liens interindividuels, mais elle est également le lieu d'une transformation qui va modifier les paramètres et les structures de la communauté ${ }^{6}$.
\end{abstract}

Selon un profil social, la notion de performance est symptomatique de cette irrépressible dualité : entre le mouvement (changement, innovation, surprise) et l'immobilité (institutionnalisation, consuétude, fixation). D'un point de vue social, ontologique et de théorie de la connaissance, cette dialectique n'arrête pas de nous interpeller et de nous demander des réponses : cette structure composite, ontologique et culturelle à la fois, est l'un des nœuds problématiques fondamentaux de notre pensée. Nous pensons pouvoir affirmer que la tension entre mouvement et immobilité, telle qu'elle se pose en relation avec les concepts d'actuel et de virtuel, est probablement I'une des questions majeures de l'histoire des idées.

\title{
3. Le pouvoir du concept
}

Revenons aux Métamorphoses. Le mythe de Méduse, monstre qui transforme en pierre tous ceux qui le regardent, propose, lui aussi, une structure de fixation du mouvement: Méduse actualise et crée des concepts, elle transforme la virtualité mouvante du monde en statues de pierre, immobiles et actualisées. Méduse garde ce pouvoir même après que Persée lui a coupé la tête, raison pour laquelle le héros doit la tenir cachée. Pourtant, Persée se garde bien de se libérer de cette précieuse tête. Méduse constitue son pouvoir, son arme.

6. Anne Cuisset, «Le Performance Group et la performance selon Richard Schechner», dans Le réel à l'épreuve des arts. L'écran, la rue, la scène, Paris : L'Harmattan, 2007, p. 123. 
Lorsqu'il rencontre Atlas et qu'il doit combattre ce géant, c'est grâce à cette tête que Persée le vaincra. Ovide le souligne bien: Persée n'a pas la force d'Atlas, mais il lève la tête de Méduse et le géant est pétrifié?.

L'immobilité produite par Méduse apparaît, elle aussi, ambiguë et instable. En effet, Méduse est également cause de mouvement. Lorsque Persée lui coupe la tête, de son sang naissent Chrysaor - un géant - et son frère Pégase, le cheval ailé, mobilité même, rêve de mobilité absolue qui hante les hommes depuis toujours. Et cette mobilité est associée de façon structurelle à l'immobilité, à la pétrification.

La suite de l'histoire de Méduse nous suggère encore cette association. Lorsque Persée pose la tête de Méduse sur une place et qu'il la couvre d'arbustes, les tiges sont pétrifiées et se transforment en pierre. Le processus de pétrification touche aussi les végétaux: tout ce qui est dans le mouvement se cristallise au contact de Méduse. Mais lorsque ces tiges pétrifiées sont jetées dans l'eau, elles deviennent, nous dit Ovide, la source féconde du corail. Les tiges, vivantes et en mouvement dans l'eau, deviennent pierres à l'extérieur de l'eau.

L'immobilité, la pierre, peut être la source du mouvement. Le concept, dans sa fixité, peut être le commencement du mouvement. Et ainsi, l'actuel peut être la source des dynamiques du virtuel. Une articulation complexe lie le mouvement et le repos : c'est l'oscillation qui structure le réel. II faut donc essayer de penser ce point de contact entre l'immobilité et le mouvement, cet interstice étrange. Platon l'appelle exaiphnes : l'instant ${ }^{8}$. II s'agit d'un temps sans étendue, qui arrive subitement. «L'in-stant (in-stans, en latin) c'est ce qui ne tient pas, ce qui n'est pas stable», rappelle François Jullien. À l'opposé, le «"maintenant" : main-tenant, manu tenere, ce que je tiens avec la main, ce que je maintiens et veux retenir $»^{9}$. Ce point

7. Ovide. Métamorphoses, op. cit., 4.770.

8. Cf. Parménide. 155e4-157b5. En ligne : http://data.perseus.org/citations/ urn:cts:greekLit:tlg0059.tlg009.perseus-grc1:155e

9. François JULLIEN, Faisons face au déroulement face au devancement, dans J. BIRNBAUM (dir.), Où est passé le temps?, Paris : Folio/Gallimard, 2012, p. 249 
de contact mystérieux partage sa structure avec le concept qui, lui aussi, crée un pont entre l'immobilité et le mouvement.

La difficulté de penser le passage de l'immobilité au mouvement rend ce changement particulièrement fascinant. Nous savons que, pour maîtriser le monde, pour saisir le réel, pour en comprendre le secret, nous devons saisir l'exaiphnes et maîtriser le mystère du passage du repos au mouvement. C'est ce qui fait probablement l'intérêt du mouvement technique.

\section{La merveille du mouvement}

En janvier 1896, un public étonné et, paraît-il, épouvanté ${ }^{10}$ assistait à la première projection d'un film qui est depuis resté gravé dans l'imaginaire collectif : L'Arrivée d'un train en gare de La Ciotat. Ce film a tant marqué les esprits qu'on a envie de le considérer comme le premier film de l'histoire du cinéma, même si, en réalité, il n'a pas été projeté lors de la première séance de cinéma des frères Lumière, en décembre 1895, mais seulement un mois plus tard.

Pourquoi ce film a-t-il laissé une trace si profonde? Quelles sont les caractéristiques de cette pellicule qui lui font se démarquer des autres films tournés dans la même période par les frères Lumière? Comment expliquer le charme irrésistible dégagé par ces quelques photogrammes? Comme le soutient Jean-Louis Comolli dans Corps et cadre ${ }^{11}$, «[c]ette peur exprimée par les premiers spectateurs de la séance inaugurale du Grand Café était moins une peur devant la menace du monde (l'irruption d'une locomotive) que devant la puissance même de la représentation $»^{12}$.

10. On sait que nous restons ici dans le domaine du mythe - même s'il s'agit d'une mythologie plus proche. Jean-Pierre Sirois-Trahan a montré que le récit de cet événement a été presque complètement inventé par les médias de l'époque, cf. "Mythes et limites du train-qui-fonce-sur-les-spectateurs », Limina. Le Soglie del Film/Film's Thresholds, Veronica Innocenti et Valentina Re (dir.), Udine : Forum, 2004, p. 203-221.

11. Jean-Louis COMOLLI, Corps et cadre. Cinéma, éthique, politique, Lagrasse: Verdier, 2012, p. 42.

12. J.-L. COMOLLI, Corps et cadre. Cinéma, éthique, politique, Lagrasse: Verdier, 2012, p. 42 
Le train a quelque chose de particulier qui nous fascine. Et ce quelque chose est ce qui engendre également la fascination pour le cinéma, pour les écrans en général et, finalement, pour Internet: ce je ne sais quoi consiste en le mystère de l'exaiphnes, qui semble être révélé dans le mouvement technique ${ }^{13}$.

Mais qu'est-ce qui fascine dans le mouvement technique? Le fait que nous pouvons facilement le gérer. La réalité a quelque chose qui nous échappe, justement parce qu'elle est en mouvement. Mais quand ce mouvement est produit par une machine, nous

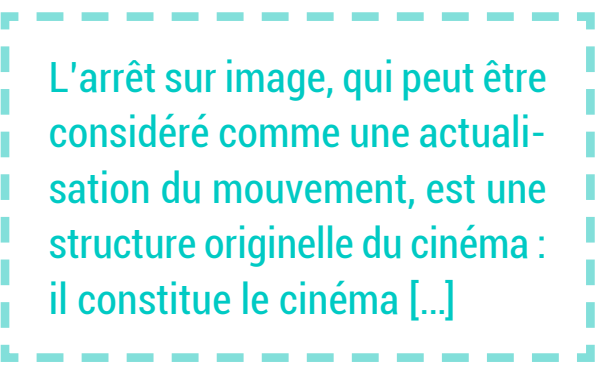
sommes en mesure de le gérer complètement. Le mouvement technique nous donne une prise sur le réel. En une phrase : ce qui distingue la scène d'un film d'une scène réelle

est que, lorsqu'on regarde un film, on peut toujours arrêter l'image et qu'en même temps, lorsque l'image est arrêtée, on peut toujours la remettre dans le flux du mouvement.

L'articulation complexe du mouvement et de l'immobilité et la difficulté à penser le point de contact entre les deux peuvent être interprétées comme une incommensurabilité entre le flux virtuel du réel et le photogramme immobile et actualisé du concept. Cette incommensurabilité est évidemment liée à la question mathématique du rapport entre discret et continu, question sur laquelle se fondaient déjà les paradoxes de Zénon. Le mouvement du réel est continu, tandis que notre façon de penser le monde et de le décrire est discrète. Si l'on analyse avec notre langage le continu du mouvement du réel, on tombe dans des paradoxes comme celui d'Achille et la tortue.

13. Pour approfondir cette thématique, cf. Marcello VITALI-ROSATI, «Le train est l'ancêtre d'Internet», 2012. En ligne: http://www.ina-expert. com/e-dossier-de-I-audiovisuel-sciences-humaines-et-sociales-et-patrimoinenumerique/le-train-est-I-ancetre-d-internet.html 
Bien évidemment, les paradoxes ont trouvé à travers les siècles plusieurs résolutions, dont celle de l'analyse mathématique proposée par Leibniz. Mais les résolutions trouvées ne diminuent en rien l'importance du problème : le continu ne peut pas être mis en relation avec le discret; si notre langage est discret, il ne peut pas décrire le continu. Plus encore, puisque notre science se base sur notre langage et que le réel, lui, est caractérisé par le continu, il y a quelque chose du réel qui échappe à la science. II ne nous reste qu'une manière pour avoir plus de prise sur la réalité : rendre discret le continu. C'est exactement ce qui se produit dans le cas du mouvement technique.

Ce qui nous fascine dans le mouvement du train comme dans celui du cinéma repose sur le fait qu'il s'agit bien de mouvements, qu'ils nous donnent l'impression du mouvement du réel et qu'en même temps, on est capable de les gérer : il dépend de nous. Le plaisir immense qu'on éprouve en regardant une scène de film dérive du fait qu'elle nous propose un mouvement qui semble identique à celui du réel, mais qu'on a toujours conscience de la possibilité de l'arrêt sur image. En ce sens, l'arrêt sur image, qui peut être considéré comme une actualisation du mouvement, est une structure originelle du cinéma : il constitue le cinéma.

«Voir le mouvement s'interrompre, c'est encore et toujours oublier que la projection, elle, continue, qu'elle doit précisément ne pas s'interrompre pour nous livrer l'effet de cette interruption, pour la dérouler en somme devant nous»,

affirme Jean-Louis Comolli :

« [a]ccéder à la dimension analytique de la décomposition du mouvement revient ainsi, très paradoxalement, à adhérer plus que jamais à la magie synthétique du cinéma - à rafraîchir la postulation de croyance dans cette impression de réalité liée au mouvement de la vie $»^{14}$.

14. J.-L. COMOLLI, Jean-Louis. Voir et pouvoir, Lagrasse: Verdier, 2004, p. 245. 
Deleuze n'a pas donné suffisamment d'importance à la critique que Bergson faisait du mouvement du cinéma. Le philosophe de Matière et mémoire ${ }^{15}$ affirme que le mouvement du cinéma est un faux mouvement parce qu'il dépend d'une machine: le mouvement n'est pas dans l'image, mais dans le projecteur, le cinéma donnant donc seulement l'illusion du mouvement. Dans L'image-mouvement ${ }^{16}$, Deleuze avance que cette critique de Bergson n'a pas beaucoup d'intérêt parce que l'expérience du cinéma nous met face à du vrai mouvement. Mais cette approche phénoménologique oublie que la conscience de la possibilité de l'arrêt sur image est toujours présente en celui qui regarde un film. La preuve du fait que l'arrêt sur image apparaît intrinsèquement lié au dispositif cinéma est historique : c'est de la volonté de pouvoir gérer le mouvement que le cinéma naît.

Pensons en particulier aux expériences de Muybridge qui expliquent bien l'idée d'une volonté de gérer le réel. Le mouvement du réel est continu et nous n'arrivons pas à le décrire. Pour le cerner, nous devons le rendre discret. Le mouvement technique consiste ainsi en la possibilité de créer une articulation gérable entre mouvement et immobilité. La caméra de Muybridge, avec les séries de ses photogrammes, est semblable à la tête de Méduse : elle pétrifie tout ce qui l'entoure, elle transforme le mouvement en immobilité, mais en même temps elle est capable - grâce au projecteur de redonner le mouvement, comme Méduse, qui engendre Pégase ou le corail. Le cinéma est en même temps Cyparissos et Pygmalion :

«Tout arrêt sur image noue ou renoue avec la chaîne photogrammatique qui est la part décisive de la base matérielle du cinéma. La machine ensuite m'induit à transformer ces photogrammes, par définition fragments discontinus de matière, d'espace et temps, en un ensemble fusionnel, une chose mentale au rendu à peu près cohérent et lisse. La dénégation fondatrice

15. H. BERGSON, Matière et mémoire. Paris : F. Alcan, 1896.

16. G. DELEUZE, L'image-mouvement. Cinéma 1. Paris : Éditions de Minuit, 1983. 
du spectateur - le cinéma n'est pas la vie, mais... pourrait s'énoncer d'une autre façon: ce ne sont que des photogrammes, mais j'y vois la vie même ${ }^{17}$.

\section{Le mouvement d'Internet}

Les changements produits par les technologies numériques constituent le point de contact entre des structures conceptuelles qui ont parcouru notre tradition culturelle et leur expression aujourd'hui. Concentrons-nous en particulier sur Internet pour comprendre la manière dont il se place dans cette ligne qui relie le mouvement du train à celui du cinéma. Commençons par constater que la première caractéristique d'Internet est précisément le mouvement. L'adjectif « virtuel » a été utilisé pour décrire les technologies numériques justement à cause de cette caractéristique. En effet, c'est le mouvement qui le distingue des médias traditionnels : Internet est un flux de données, ce qui implique que les contenus du web ne sont jamais stables ou arrêtés, à l'opposé des contenus des autres médias.

Il faut préciser le type de mouvement dont il s'agit. Internet est un flux de données qui circulent d'un point à l'autre à travers des voies: les câbles qui constituent le réseau. Voilà une première analogie avec le train : le réseau constituant Internet ressemble dans un premier temps au réseau ferroviaire. Le mouvement se déploie sur des chemins préconstitués et il est donc facilement contrôlable.

La deuxième analogie d'Internet avec le train et le cinéma repose sur le fait qu'il est numérique. Le numérique apparaît, en effet, la manifestation la plus évidente du mensonge du mouvement, qui consiste à discrétiser le continu pour pouvoir le gérer tout en gardant l'illusion du continu. En d'autres termes, le numérique s'appuie sur le même principe de discrétisation du réel que celui des vingt-quatre photogrammes par seconde du cinéma. 
Comme on le sait, le numérique est la représentation du réel à travers des nombres naturels en base deux, c'est-à-dire en une série de 0 et de 1. Pour que cette représentation soit possible, il faut préalablement échantillonner le réel.

Le numérique nous donne l'impression du continu, mais il est discret; il nous donne l'impression du mouvement réel, mais il est un mouvement technique. Ce qui nous passionne dans le numérique, c'est qu'il nous donne l'illusion du mouvement et qu'en plus nous pouvons facilement le gérer, le contrôler, le décrire.

La troisième caractéristique du mouvement d'Internet consiste évidemment en le fait qu'il est produit et géré par une machine. Cet aspect, complètement banal, est souvent oublié dans le cas particulier du web : nous avons tendance à négliger le fait que les contenus du web et leur mouvement, leur agencement comme leur affichage, dépendent en premier lieu des machines. Ce sont, par exemple, les algorithmes des moteurs de recherche qui déterminent la hiérarchie des contenus et qui la font varier dans le temps. Le code relie des pages à des bases de données, permet et détermine la mise à jour des plateformes de publication ; le code programme le navigateur générant l'affichage et le mouvement des informations sur l'écran.

Internet agit donc comme un mouvement de données sur des voies, semblable aux trains sur les chemins de fer, discret mais donnant l'impression du continu, et semblable au cinéma, géré par une machine et donc facile à contrôler.

\section{L'entre-temps de l'action numérique: entre le direct et l'enregistrement}

Regardons maintenant la façon dont ce mouvement s'articule et se décline dans les pratiques numériques les plus courantes. En se penchant sur l'expérience que l'usager fait de et dans I'hypertexte, Christian Vandendorpe soutient que

«[l]e programme peut aussi occulter des mots importants sous un cache, afin de susciter la curiosité du 
lecteur et de tenir en éveil son attention: pour faire disparaître ce cache, il suffira, par exemple, d'en approcher la souris. On définira cette interactivité comme la capacité pour l'usager de créer des événements sur la page ${ }^{18}$.

Les icônes, les couleurs, les effets sonores, certains escamotages cinématographiques (Vandendorpe cite le fondu au noir, le fondu enchaîné, le mouvement en spirale, l'effet de puzzle), l'indexation des contenus par les algorithmes, concourent à produire ce que l'auteur nomme « hyperlive»: les conditions du live, de la performance, de l'événement, de la présence, s'organisent comme un ensemble procédural, sensoriel et sémiotique capable d'émanciper l'écran de son histoire de surface de représentation pour en faire finalement un espace de mouvement et d'action. L'événement, c'est aussi un avènement et une éventualité : evenire, en latin, signifie «sortir», «se produire», «advenir»; l'événement est ce qui survient, ce qui peut éventuellement arriver, ce qui est en train de se passer, et enfin, seulement après, ce qui s'est passé. Une autre acception du mot consiste en ce que la notion d'éventualité a en droit, où l'on indique par là ce qui ne peut entrer en vigueur sans que certaines conditions se trouvent remplies. Dans le web, l'ensemble des conditions de nos actions procèdent du design, des fonctions et des algorithmes qui caractérisent les différentes plateformes. Si l'on observe les applications de messagerie multimédia comme WhatsApp ou Messenger, on constate qu'elles sont en train de suggérer et de développer une action qui, pour l'usager, représente l'expérience du temps en mouvement, le temps en train (encore le train !). Allant dans la direction de ce que Yves Citton appelle la «fascination du suspense gestuel ${ }^{19}$, le graphisme exploite des signes de ponctuation pour exprimer une aptitude temporelle bien particulière. Ainsi, les points de suspension indiquant l'ellipse deviennent le symbole d'une attente qui nous affecte

18. Christian VANDENDORPE, Du papyrus à l'hypertexte, Paris : Éditions La Découverte, 1999, p. 203.

19. Yves CITTON, L'écologie de l'attention, Paris : Seuil, 2014, p. 149. 
et nous attire: ces trois points pétillent dans un petit ovale pendant que notre interlocuteur - c'est du moins ce que nous pouvons lire à côté de son avatar - est en train d'écrire ou d'enregistrer (dans le cas d'un message audio). Cette attente en commun est ce qu'on appelle «temps réel », réel parce qu'identique pour nous et notre interlocuteur. II s'agit d'un temps induit et géré par la machine qui se charge de médier entre nous deux, un temps technique, donc en réalité une contrainte, une inconvenance même, temps dont le serveur central a besoin pour envoyer sur un périphérique le message reçu de l'autre. Suspens, attente, mouvement, live : ce sont des dimensions de plus en plus normées dans les réseaux sociaux et qui modifient radicalement le fonctionnement des notifications. Sur Snapchat, depuis quelques mois, on reçoit un message nous informant du fait que quelqu'un est en train de, juste en train de... nous envoyer un snap. Sur Facebook, contrairement à auparavant, on peut aujourd'hui savoir que quelqu'un est en train d'écrire un commentaire sur notre mur (ou sur celui d'un ami où nous avons aussi écrit), tout comme on peut «être en direct » par l'intermédiaire d'une captation vidéo. Avant un fragment identitaire et temporel inexorablement passé, la photo de profil peut maintenant être prise au moment même grâce à la caméra Facebook, ou être une vidéo captée au préalable. Le design et les fonctions des interfaces semblent s'orienter de plus en plus vers la prise sur le temps en mouvement, vers le côté fluide de la performance, vers le mouvement virtuel, et pourtant un aspect fondamental de la performativité numérique demeure celui de l'enregistrement - et donc de l'actualisation, notamment dans la forme de la notification.

Lisons une succession quelconque de notifications Facebook: « Julien a ajouté une photo » à l'instant; «Vous avez partagé pendant 3 jours d'affilée et vos amis réagissent » il y a 20 minutes; « Nina Tyler a actualisé son statut » il y a 45 minutes; " C'est l'anniversaire de Arnaud Maïsetti et 3 autres personnes aujourd'hui. Fêtez ça avec eux » il y a une heure; "Michael Sinatra a répondu à votre commentaire sur sa vidéo » hier à 19 h 40 ; «Sara-Ananda Badger a commenté votre publication » vendredi à 19 h ; « David Desrimais a publié 
du contenu pour la première fois depuis un certain temps » vendredi à 11 h 30 ; " Ilaria Cascasi a réagi à une publication » mercredi à 23 h 44 ; « Maximilien Gaudette et 36 autres personnes ont récemment aimé votre photo » mardi à $11 \mathrm{~h} 59$; «Clara Mayer a actualisé son statut » mardi à 22 h 30 ; « Denis Chomel était en direct » lundi à 12 h. ${ }^{20}$

Sur Facebook, le rouleau de notifications est une chronologie de plus en plus détaillée et minutieuse à laquelle n'échappe aucun des micro-gestes accomplis par nos amis Facebook. Lorsqu'ils partagent ou aiment ou publient quelque chose, ou y réagissent, nous recevons des notifications nous avertissant de ce qui vient d'être fait. On peut choisir de recevoir ou non des notifications Facebook au sujet de ses amis proches ou de leur anniversaire, on peut choisir que soit émis ou non un son à la réception de ces notifications, mais ainsi qu'on peut le lire sur la page consacrée à leurs paramètres, on ne peut pas les désactiver tout court. Le rythme des notifications Facebook, lesquelles par défaut, au fil de ces dernières années, a augmenté en fréquence et en nombre, constitue la structure temporelle essentielle du réseau social le plus important.

"Le design des notifications est marqué par une tension croissante entre deux orientations normatives relatives respectivement à la conduite de l'action en plan et aux exigences de réactivité et de disponibilité. Le statut ambivalent des interruptions reflète cette tension: perturbantes (par rapport à l'exécution en plan) ou positives (comme occasion de réactivité) » ${ }^{21}$.

Dans l'ensemble du rouleau chronologique où elles sont listées au fur et à mesure, les notifications s'agencent en une narration située dans un lieu particulier. La narration résultant de l'enchaînement de plusieurs notifications s'inscrit dans un sous-environnement auquel on accède en cliquant sur une

20. Suite de notifications du compte Facebook de Peppe Cavallari (20/06/2017).

21. Christian LICOPPE, «Pragmatique de la notification», Traces, 16/2009. 
icône représentant la planète : ce lieu, qui a la forme d'un rouleau que nous pouvons faire défiler vers le bas comme vers le haut, est un endroit privé accessible seulement par l'utilisateur.

«Par définition, la notification advient, apparaît, surgit, et elle occasionne potentiellement des interruptions. Les dispositifs des notifications formatent le type d'interruptions auxquelles ils donnent lieu. Ils sont le siège d'une tension entre concentration et dispersion, entre pilotage interne de l'activité individuelle... et pilotage externe » (Ibid.).

Cette interruption intéresse notre réflexion sur le rapport entre actuel et virtuel, entre immobilité et mouvement : en effet, la notification arrive très souvent pendant qu'on est en train de lire, d'écrire, ou d'écouter quelque chose. Elle fait irruption en nous obligeant d'abord à remanier notre écran, ensuite à renégocier les conditions de notre regard, de nos ré-actions et de notre attention. Interrompre le flux de notre action constitue un important moment performatif, entendu au sens de surprise, de changement de scène, de passage à autre chose. Le temps dans lequel on baigne lorsqu'on est en ligne et joignable sur plusieurs plateformes est celui d'une continuité d'interruptions - des interruptions tellement continues et enchaînées les unes aux autres qu'elles forment une unité spatiotemporelle. On retrouve encore une fois la relation complexe entre discret et continu. Les interruptions discrètes, comme les vingt-quatre photogrammes par seconde, permettent l'illusion du mouvement continu. «La notion même d'interruption devient ambivalente. Cette ambivalence est renforcée par la prise de conscience que beaucoup d'interruptions sont liées à des événements de communication inaugurés par des sollicitations d'autrui. » ${ }^{22}$

«C'est ce tissu d'événements incessants de notification qui est le creuset de la rencontre (quelqu'un est passé sur votre site, vous a fait un clin d'œil, vous a envoyé un message, etc., dans le cas des sites de 
rencontre amoureuse). Ce sont les notifications de tous les microévénements concernant ses membres qui rendent visibles l'activité du réseau social et, in fine, le réseau social lui-même dans Facebook. La notification est devenue une commodité, produite et consommée en masse $»^{23}$.

L'actuel, qui ici se concrétise dans la forme de l'enregistrement, devient la condition de possibilité du mouvement. Une notification est immanquablement associée à un marqueur temporel: « il y a X minutes», «hier à 23 h 40 », « 27 juin à $16 \mathrm{~h} 27$ » : dans la boîte de nos mails, dans les applications de messagerie multimédia, sur le mur Facebook, comme dans le rouleau des notifications et dans le fil d'actualité, une chronologie indéfectible se déroule. Récemment, l'interface de Facebook a ajouté un bouton de plus sur notre mur, celui de notre historique personnel, où nous pouvons voir la liste exacte de tous nos faits et gestes banals, y compris nosj'aime et nos réactions. Notre action est immédiatement enregistrée et répertoriée, listée et collectée dans des box qu'on peut consulter à tout moment : c'est la cristallisation du temps propre à cette technologie de l'enregistrement qu'est le numérique. Comme sur Snapchat, on reçoit des notifications nous informant que I'un de nos contacts est en train de nous envoyer un snap on pourrait parler de notifications des intentions; recevoir une notification, sur Facebook, signifie encore que quelque chose vient de se passer et qu'elle s'actualise en se marquant temporellement, afin de pouvoir s'inscrire dans une histoire. Cette dernière devient le contexte de toutes nos actions : ce que nous faisons prend place dans ce registre, et notre action y trouve son sens en arrivant avant ou après quelque chose d'autre. Sans cette histoire, nous serions complètement dépaysés, frappés d'une amnésie ne pouvant qu'affaiblir l'intensité et la pertinence de nos échanges. Les marqueurs temporels ainsi que la permanence des messages dans la messagerie nous permettent d'avoir des relations continues, de pouvoir consulter un message, le dernier, datant peut-être d'il y a quelques mois, de nous aider à retrouver le sens d'un dialogue, de tisser l'intrigue d'un discours qui ne nous échappe pas. 


\section{Conclusion}

Ce jeu entre mouvement et immobilité qui caractérise si profondément les environnements numériques nous montre que l'actuel peut devenir la condition de possibilité du mouvement. II n'y a de mouvement que parce qu'il y a des interruptions. La suite des interruptions fait la continuité du « temps réel ». Comme dans les Métamorphoses d'Ovide, le monde numérique est fait d'innombrables itérations du passage entre mouvement et immobilité. L'infinie poursuite de la haute qualité ou de la haute définition pourrait s'expliquer ainsi : plus fin est l'échantillon, plus nombreuses sont les actualisations, et plus puissante sera l'illusion du mouvement. L'illusion, avons-nous dit... Ou alors, finalement, dans un renversement inattendu, le mouvement ne serait-il que cette illusion?

Qu'on se rappelle Melville et l'idée selon laquelle il serait impossible de produire une image fiable de la baleine: son mouvement la rend insaisissable, et tout arrêt sur image ne serait qu'une trahison de l'original. L'actuel apparaît bien comme un contrecoup du mouvement virtuel. Mais si l'on voulait vraiment saisir ce mouvement, on risquerait de perdre notre vie : le mouvement implique à nouveau l'arrêt - cette fois définitif :

For all these reasons, then, any way you may look at it, you must needs conclude that the great Leviathan is that one creature in the world which must remain unpainted to the last. True, one portrait may hit the mark much nearer than another, but none can hit it with any very considerable degree of exactness. So there is no earthly way of finding out precisely what the whale really looks like. And the only mode in which you can derive even a tolerable idea of his living contour, is by going a whaling yourself; but by so doing, you run no small risk of being eternally stove and sunk by him. ${ }^{24}$

24. Herman MELVILLE, «Chapter 55: Of the Monstrous Pictures of Whales », Moby Dick; or, The Whale, 5, p. 410. En ligne: http://www.planetebook.com/ ebooks/Moby-Dick.pdf. 
BERgSON, Henri, L'évolution créatrice, Paris, Presses universitaires de France, 1966.

- Matière et mémoire, Paris, F. Alcan, 1896.

Citton, Yves, L'écologie de l'attention, Paris, Seuil, 2014.

ComolLI, Jean-Louis, Corps et cadre, Lagrasse, Verdier, 2012.

- Voir et pouvoir, Lagrasse, Verdier, 2004.

CUISSET, Anne, «Le Performance Group et la performance selon Richard Schechner », dans Le réel à l'épreuve des arts. L'écran, la rue, la scène, Paris, L'Harmattan, 2007.

Deleuze, Gilles, L'image-mouvement. Cinéma 1, Paris, Éditions de Minuit, 1983.

- Différence et répétition, Paris, France, Presses universitaires de France, 1972.

JULLIEN, François, "Faisons face au déroulement face au devancement ", dans Birnbaum J. (dir.), Où est passé le temps ?, Paris, Folio-Gallimard, 2012.

LÉvy, Pierre, Qu'est-ce que le virtuel ?, Paris, Éditions La Découverte, 1995. LICOPPE, Christian, « Pragmatique de la notification », Traces, 16/2009.

OVIDE, Les Métamorphoses, traduction (légèrement adaptée) de G.T. Villenave, Paris, 1806. http://bcs.fltr.ucl.ac.be/META/01.htm

Sirois-TrahaN, Jean-Pierre, "Mythes et limites du train qui fonce sur les spectateurs », Limina. Le Soglie del Film/Film's Thresholds, Veronica Innocenti et Valentina Re (dir.), Udine, Forum, 2004, pp. 203-221.

VANDENDORPE, Christian, Du papyrus à I'hypertexte, Paris, Éditions La Découverte, 1999.

VITALI-RosatI, Marcello, «Le train est l'ancêtre d'Internet », 2012. http:// www.ina-expert.com/e-dossier-de-l-audiovisuel-sciences-humaines-etsociales-et-patrimoine-numerique/le-train-est-I-ancetre-d-internet.html

- S'orienter dans le virtuel, Paris, Hermann, 2012.

WuLF, Christoph, Une anthropologie historique et culturelle. Rituels, mimésis sociale et performativité, trad. fr., Paris, Téraèdre, 2007. 